J. Gynäkol. Endokrinol. AT 2020 · 30:138-143 https://doi.org/10.1007/s41974-020-00146-8 Online publiziert: 20. August 2020

(c) Der/die Autor(en) 2020

\section{Katharina Feil · Bettina Toth}

Universitätsklinik für Gynäkologische Endokrinologie und Reproduktionsmedizin, Medizinische Universität Innsbruck, Innsbruck, Österreich

\title{
Die Transgendersprechstunde - was gibt es zu beachten?
}

\section{Innsbrucker Erfahrungen anhand von Fallbeispielen}

\section{Transgender Center Innsbruck}

Seit der Gründung der Universitätsklinik für Gynäkologische Endokrinologie und Reproduktionsmedizin an der Medizinischen Universität Innsbruck im Jahr 2003 werden hier Jugendliche und Erwachsene mit Vorliegen einer Geschlechtsinkongruenz betreut. Die interdisziplinäre Vernetzung mit den Universitätskliniken für Gynäkologie und Geburtshilfe, Pädiatrie, Psychiatrie, Psychiatrie im Kindes- und Jugendalter, Hör-, Stimmund Sprachstörungen, plastische Chirurgie und Urologie sowie einem juristischen Beirat konnte in den letzten Jahren immer weiter ausgebaut werden. 2016 wurde gemeinsam mit den aufgeführten Fachrichtungen das erste Transgendercenter Österreichs gegründet. Derzeit befinden sich ungefähr $200 \mathrm{Trans}^{\star}$ Personen in unserer Betreuung, davon war etwa ein Drittel bei Erstvorstellung minderjährig. Einmal im Monat treffen sich die beteiligten Disziplinen zum TransgenderBoard und erarbeiten ein individuelles Behandlungskonzept. Durch diese enge Vernetzung können die Behandlungsabläufe für Trans ${ }^{\star}$ Personen optimiert und die einzelnen therapeutischen Angebote niedrigschwellig umgesetzt werden.

Der Erstkontakt am Transgender Center Innsbruck erfolgt meist entweder über die Transgendersprechstunde der gynäkologischen Endokrinologie und Reproduktionsmedizin oder im Fall von Erwachsenen über die sexualmedizinische Ambulanz der Psychiatrie bzw. im Fall von Kindern oder Jugendlichen über die kinder- und jugendpsychiatrische Spezialsprechstunde.

\section{Begriffsklärung}

Die Diskrepanz zwischen dem biologischen, bei Geburt zugeordneten Geschlecht und dem erlebten Geschlecht wird durch den Begriff "Transgender“ beschrieben. „Cisgender" bezeichnet im Gegensatz dazu eine Person, bei der biologisches und erlebtes Geschlecht übereinstimmen. Der Begriff Geschlechtsdysphorie wird verwendet, wenn die oben genannte Diskrepanz zu einem Leidensdruck führt. Häufig sind medizinische Behandlungsoptionen wie eine Hormontherapie oder chirurgische Intervention von den Betroffenen erwünscht und führen $\mathrm{zu}$ einer Besserung der Geschlechtsdysphorie. Jedoch schließt eine Therapie nicht zwangsläufig körpermodifizierende Maßnahmen ein. Das Spektrum der Geschlechtlichkeit ist vielfältig: Sowohl eine eindeutig weibliche oder männliche Geschlechtsidentität im Sinne eines binären Geschlechtersystems als auch eine nonbinäre bzw. "gender non-conforming" Geschlechtsidentität kann angestrebt werden.

Die zunehmende mediale Präsenz der Thematik trägt entscheidend zur Enttabuisierung bei. Dies spiegelt auch die neue Klassifikation in der ICD-11 wider, welche im Mai 2019 verabschiedet wurde und ab 2022 in Kraft treten soll [1]. Während in der ICD-10 noch der Begriff „Transsexualismus“ verwendet wird, wird in der ICD-11 unter „conditions related to sexual health“ die Geschlechts- inkongruenz (GI) zu finden sein. So soll entscheidend zur Entpathologisierung beigetragen werden.

\section{Epidemiologie}

Es ist schwierig, die genaue Prävalenz und Inzidenz der Geschlechtsinkongruenz zu erheben. In einer Metaanalyse zeigten Collin et al. 2016 eine Prävalenz von 5,8/100.000 für Male-to-female(MTF)und 2,5/100.000 für Female-tomale(FTM)-Transgender in Abhängigkeit von transgenderspezifischen Diagnosen [2]. Die Prävalenz in Abhängigkeit von geschlechtsangleichenden Maßnahmen lag in dieser Studie mit 9,2/100.000 noch deutlich höher. Zudem lässt sich eine Zunahme der GI unter Jugendlichen in den letzten Jahren beobachten, wobei vor allem gehäuft Trans ${ }^{\star}$ Jungen (FTM) vorstellig werden $[3,4]$.

\section{Fallbeispiel 1}

Katja G., 12 Jahre alt, wird mit ihrer Mutter in unserer Sprechstunde mit dem Wunsch nach Pubertätsarretierung vorstellig. Bereits im Vorfeld wurde eine Abklärung an der Kinder- und Jugendpsychiatrie gestartet und ist zum Zeitpunkt der Erstvorstellung bei uns noch im Gange. Katja fühlt sich bereits seit frühester Kindheit als Junge, der Wunschname ist Max. Bei Max hat vor circa 2 Monaten die Thelarche eingesetzt, aktuell ist die Brustentwicklung bei Tanner 2. Das Knochenalter beträgt 13 Jahre +2 Monate, die Knochendichte liegt im Normbereich 
und die Menarche hat noch nicht stattgefunden. Nach Rücksprache mit den KollegInnen von der Kinder- und Jugendpsychiatrie wird die Diagnostik vorangetrieben, um zeitnah eine Pubertätsarretierung zu starten.

\section{Diagnostik}

Der diagnostische Prozess soll eine psychiatrische, psychotherapeutische und psychologische Beurteilung umfassen. Im Diagnostischen und Statistischen Manual Psychischer Störungen (DSMV) der American Psychiatric Association wird Geschlechtsdysphorie als klinisch relevanter Leidensdruck durch die Diskrepanz zwischen Zuweisungsgeschlecht und erlebtem Geschlecht definiert [5].

Vor einer körpermodifizierenden Therapie ist ein Konsensbeschluss bzw. eine positive Stellungnahme des fallführenden Psychiaters eine Grundvoraussetzung. Für eine Personenstandsund Namensänderung wird ebenso wie für eine gegengeschlechtliche Hormontherapie eine Stellungnahme gefordert. Zur Planung operativer Maßnahmen ist eine zweite positive Stellungnahme erforderlich. Alle Stellungnahmen sind 2 Jahre gültig. Ohne Vorliegen der schriftlichen Stellungnahmen von dafür qualifizierten KollegInnen wird vom Einsatz jeglicher geschlechtsangleichender Therapien dringend abgeraten. Eine interdisziplinäre Betreuung ist daher eine notwendige Voraussetzung.

In Österreich gelten die Empfehlungen des Gesundheitsministeriums für den Behandlungsprozess bei $\mathrm{Ge}$ schlechtsdysphorie bzw. Transsexualismus, welche zuletzt 2017 überarbeitet wurden [6]. Diese Empfehlungen basieren zu großen Teilen auf den Versorgungsempfehlungen für die Gesundheit von transsexuellen, Transgender- und geschlechtsnichtkonformen Personen der World Professional Association for Transgender Health (WPATH; [7]).

\section{Pubertätsarretierung mittels GnRH-Analoga}

Wie die Gabe von GnRH-Analoga (GnRH-a) ist auch der Einsatz von Sexualsteroiden bei Trans ${ }^{\star}$ Personen ein „off label use“, worüber die PatientInnen bzw. im Falle von Jugendlichen auch die Erziehungsberechtigten ausreichend aufgeklärt werden müssen. Bei Jugendlichen sollte frühestens ab Einsetzen der Pubertät (Tanner 2) mit einer GnRH-a-Therapie begonnen werden. Häufig verwendete Präparate sind unter anderem Triptorelin 3,75 mg (Verabreichung alle 4 Wochen i.m. oder s.c.), Triptorelin 11,25 mg (Verabreichung alle 12 Wochen i.m.) oder Leuprorelinacetat $3,75 \mathrm{mg}$ (Verabreichung alle 4 Wochen s.c.). Häufige Nebenwirkungen sind Libidoverlust, eine rasche Abnahme der morgendlichen Erektionen bei MTF und Hitzewallungen bei FTM. Gemäß natio- nalen und internationalen Richtlinien wird der Start mit der gegengeschlechtlichen Hormontherapie ab einem Alter von circa 16 Jahren empfohlen und muss individuell diskutiert werden $[8,9]$.

Bei Pubertätsbeginn sollte die Bestimmung des Knochenalters mittels einer Röntgenaufnahme der linken Hand erfolgen [3, 10]. Zudem sollte eine Knochendichtemessung veranlasst werden, insbesondere bei einer geplanten pubertätsunterdrückenden Therapie mittels GnRH-a [3, 8]. Vor allem die Anwendung von GnRH-a, aber auch die langjährige Anwendung von CHT kann zu einem Verlust von Knochendichte führen $[11,12]$.
Hier steht eine Anzeige.

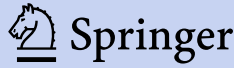




\section{Fallbeispiel 2}

Der 18-jährige Mark (zuvor Aline), ein junger Trans ${ }^{\star}$ Mann, wird mit positiven Stellungnahmen der betreuenden Psychotherapeutin und eines Psychiaters sowie mit internistischen Voruntersuchungen in unserer Sprechstunde vorstellig und wünscht den Start einer gegengeschlechtlichen Hormontherapie. Auf eine gynäkologische Untersuchung wurde verzichtet, da der Patient Virgo ist. Eine Fertilitätsprotektion wünscht Mark aktuell nicht. Da bereits alle notwendigen Abklärungen durchgeführt wurden, können wir direkt mit der Testosterontherapie starten. Zusätzlich rezeptieren wir Mark eine Gestagen-only-Pille, um die für ihn sehr belastende Periodenblutung zu unterdrücken.

\section{Hormontherapie}

Neben der oben beschriebenen Diagnostik von psychiatrischer, psychologischer und psychotherapeutischer Seite wird eine somatische Abklärung möglicher Kontraindikationen in die Wege geleitet. $\mathrm{Zu}$ Kontraindikationen für eine Östrogentherapie zählen östrogensensitive Neoplasmen, chronische Lebererkrankungen und Thromboembolien (bei oraler Einnahme der Östrogene; [7]). Bei einer geplanten Testosterontherapie zählen eine bestehende Schwangerschaft und eine instabile Angina pectoris sowie bedingt auch östrogensensitive Neoplasmen als Kontraindikationen. Die in - Tab. 1 dargestellten Erkrankungen können mit einer CHT assoziiert sein und sollten daher vor Start der CHT abgeklärt werden. Die initiale Bestimmung von LH, FSH, Östradiol, Testosteron, 25-OHVitamin D, des Eisenstatus, der Lipide, des Blutbilds sowie der Leber- und Nierenfunktionsparameter empfiehlt sich bei ansonsten gesunden Jugendlichen und Erwachsenen [3, 8].

Die gegengeschlechtliche Hormontherapie bei MTF erfolgt mit Östrogenen in der Form von transdermalem $17 \beta$ Östradiol oder Östradiolhemihydrat oral. Die empfohlene Dosierung liegt bei $50-200 \mu \mathrm{g} 17 \beta$-Östradiol transdermal bzw. 1,5-3 mg Östradiolhemihydrat oral. Bei Jugendlichen sollte, vor allem

J. Gynäkol. Endokrinol. AT 2020 • 30:138-143 https://doi.org/10.1007/s41974-020-00146-8

c) Der/die Autor(en) 2020

\section{K. Feil · B. Toth \\ Die Transgendersprechstunde - was gibt es zu beachten? Innsbrucker Erfahrungen anhand von Fallbeispielen}

\begin{abstract}
Zusammenfassung
Aufgrund einer stetigen Zunahme der Personen mit Geschlechtsinkongruenz bzw. dem Wunsch nach geschlechtsangleichenden Therapien ist eine Auseinandersetzung mit diesem Thema für alle ÄrztInnen ebenso wie für das medizinische Personal von wachsender Dringlichkeit. Die Initiierung der therapeutischen Maßnahmen sollte idealerweise in spezialisierten Zentren unter interdisziplinärer Abstimmung erfolgen, die Begleitung durch niedergelassene KollegInnen ist jedoch von ebenso großer Bedeutung. Die Therapiemöglichkeiten
\end{abstract}

umfassen die gegengeschlechtliche Hormontherapie, Operationen, logopädische Betreuung und psychotherapeutische sowie psychiatrische Behandlungen. Die Betreuung von Trans*Personen sollte ein Leben lang erfolgen und auch allgemeine Vorsorgeaspekte miteinschließen.

Schlüsselwörter

Transgender - Geschlechtsinkongruenz • Gegengeschlechtliche Hormontherapie · Geschlechtsangleichende Therapie · Transsexualität

\section{The Transgender Clinic: What Needs to Be Considered? Innsbruck Experiences Based on Case Studies}

\section{Abstract}

Due to a steady increase in the number of people with gender incongruence and the desire for gender-affirming therapies, dealing with this topic is of increasing importance for all physicians as well as for medical staff. The initiation of therapeutic measures should ideally take place in specialized centers under interdisciplinary coordination, but the guidance by practice-based colleagues is just as important. Therapy options include cross-sex hormone therapy, surgery, speech therapy, and psychotherapeutic and psychiatric therapies. The care of trans* people should be lifelong and should also include general screening aspects.

\section{Keywords}

Transgender $\cdot$ Gender incongruence $\cdot$ Crosssex hormone treatment - Gender-affirming therapy · Transsexuality bei noch nicht weit fortgeschrittener Pubertät, die Medikation eingeschlichen werden, mit einer Steigerung der Dosierung alle 6 Monate. Der laborchemische Zielbereich für Östradiol liegt bei $100-200 \mathrm{pg} / \mathrm{ml}$. Eine feminisierende CHT führt unter anderem $\mathrm{zu}$ Brustdrüsenwachstum, Libidoreduktion, Abnahme spontaner Erektionen, Veränderung der Fettverteilung, leichter Abnahme von Haarwuchs im Gesicht und am Körper [8]. Bei Jugendlichen kommt es zudem zu einem rascheren Verschluss der Wachstumsfugen. Dies führt $\mathrm{zu}$ einer geringeren Endgröße, welche im Bereich der Durchschnittsgröße von Cisfrauen liegt. Eine CHT hat bei bereits stattgehabtem Stimmbruch keinen Effekt auf die Stimme. Daher sollte eine logopädische Betreuung und im späteren Verlauf gegebenenfalls auch eine chirurgische Therapie angeboten werden.

$\mathrm{Da}$ Östrogene allein keine suffiziente Suppression der Androgenproduktion in den Gonaden bewirken, müssen zusätzlich antiandrogene Substanzen wie Cyproteronacetat, Spironolacton oder GnRH-a eingesetzt werden. Sollte eine Gonadektomie erwünscht sein, erfolgt ab diesem Zeitpunkt nur mehr die alleinige Östrogentherapie. Zu Cyproteronacetat ist kürzlich eine Warnung der EMA erschienen [13]. Entsprechend dieser Anwendungsbeschränkung sollte die Verschreibung von Cyproteron in der Dosis von $10 \mathrm{mg}$ täglich oder mehr aufgrund eines erhöhten Meningeomrisikos nur nach Ausschöpfen anderer Therapieoptionen erfolgen und schrittweise bis zur geringstmöglichen Dosierung reduziert werden. 


\begin{tabular}{|c|c|c|c|}
\hline \multicolumn{2}{|c|}{$\begin{array}{l}\text { MTF } \\
\text { Östrogentherapie }\end{array}$} & \multicolumn{2}{|c|}{$\begin{array}{l}\text { FTM } \\
\text { Testosterontherapie }\end{array}$} \\
\hline Hohes Risiko & Moderates Risiko & Hohes Risiko & Moderates Risiko \\
\hline \multirow{6}{*}{$\begin{array}{l}\text { Thrombo- } \\
\text { embolie }\end{array}$} & Makroprolaktinom & \multirow[t]{6}{*}{ Polyglobulie } & Leberfunktionsstörungen \\
\hline & Mammakarzinom & & Hypertension \\
\hline & KHK & & Mammakarzinom \\
\hline & $\begin{array}{l}\text { Zerebrovaskuläre Verände- } \\
\text { rungen }\end{array}$ & & KHK \\
\hline & Cholelithiasis & & Zerebrovaskuläre \\
\hline & Hypertriglyzeridämie & & Veranderungen \\
\hline
\end{tabular}

\section{Tab. 2 Verlaufskontrollen unter CHT. (Modifiziert nach [8])}

\begin{tabular}{ll}
\hline Alle 3-6 Monate im 1. Jahr, danach 1- bis 2-mal pro Jahr bei Jugendlichen \\
\hline FTM & MTF \\
\hline Gewicht & Gewicht \\
\hline Blutdruck & Blutdruck \\
\hline Tanner-Stadium & Tanner-Stadium \\
Größe & Größe \\
Testosteron & Testosteron \\
Östradiol & Östradiol \\
\hline Hämoglobin oder Hämatokrit & Prolaktin \\
\hline Lipide & Elektrolyte bei Spironolacton \\
\hline MTF "male-to-female", FTM "female-to-male" & \\
\hline
\end{tabular}

Die gegengeschlechtliche Hormontherapie bei FTM kann unter anderem mithilfe von Testosteronundecanoat (Zieldosis $1000 \mathrm{mg}$ alle 12 Wochen) oder Testosteron in Gelform (Zieldosis $1 \% 25 \mathrm{mg} / \mathrm{Tag}$ ) erfolgen. Testosteronenantat (Zieldosis beträgt $250 \mathrm{mg}$ alle 3 Wochen) kann ebenfalls eingesetzt werden, ist derzeit allerdings am österreichischen Markt nicht erhältlich. Auch bei Trans ${ }^{\star}$ Jungen muss eine einschleichende Dosierung mit anschließender schrittweiser Steigerung bzw. eine höhere Anfangsdosis je nach Pubertätsstadium individuell diskutiert werden. Bei Erwachsenen wird häufig Testosteronundecanoat eingesetzt, da dieses eine bessere Pharmakokinetik aufweist und stabile Testosteronlevel nachweisbar sind [14, 15]. Zur Anwendung im Rahmen einer Pubertätsinduktion bei Jugendlichen liegen keine Daten vor [15]. Irreversible Effekte der virilisierenden Hormontherapie sind eine Vertiefung der Stimme sowie eine Klitorishypertrophie. Zudem treten unter anderem auch Akne, Bartwuchs, vermehrte Kör-
tientInnen, die nach Gonadektomie die CHT stoppen, durchgeführt werden.

\section{Fertilitätsprotektion}

Sowohl die American Society for Reproductive Medicine als auch die Endocrine Society und die WPATH empfehlen die Aufklärung über fertilitätsprotektive Maßnahmen bereits vor Beginn der CHT und unterstreichen so ausdrücklich die Wichtigkeit der Fertilitätsprotektion für Trans ${ }^{\star}$ Personen $[7,8,16]$. In jedem Fall ist die Aufklärung über Möglichkeiten der Fertilitätsprotektion bereits vor Beginn der CHT nötig, die erwünschten Maßnahmen müssen spätestens vor einer Gonadektomie erfolgen. Speziell bei der Beratung von Jugendlichen und jungen Erwachsenen ist es wichtig, einen möglichen späteren Kinderwunsch zu thematisieren und die verschiedenen Möglichkeiten aufzuzeigen. Laut einer Studie von Chen et al. wünscht sich circa ein Drittel der Trans ${ }^{\star}$ Jugendlichen biologisch eigene Kinder [17]. Ebenso wichtig ist es auf der anderen Seite, auch über eine sichere Kontrazeption aufzuklären.

Bei MTF gibt es Hinweise darauf, dass bereits vor Start einer CHT Spermienkonzentration, Spermiengesamtzahl, Motilität und Spermien mit normaler Morphologie reduziert sind [18]. Spermiogramme unter laufender CHT scheinen zudem deutlich schlechter, jedoch nach Pausieren der CHT vergleichbar mit Spermiogrammen vor Start der CHT zu sein [19]. Nichtsdestotrotz kann eine CHT auch eine Azoospermie zur Folge haben, daher sollte Trans ${ }^{\star}$ Frauen eine Kryokonservierung von Sperma vor Start der CHT angeboten werden. Auch eine Kryokonservierung von Hodengewebe für eine spätere testikuläre Spermienextraktion (TESE) ist möglich.

Die Testosterontherapie bei FTM führt zur vorübergehenden Amenorrhö, nicht jedoch zur Depletion der Follikel. So kann nach Absetzen oder unter insuffizienter Testosterontherapie von einer Rückkehr der Fertilität ausgegangen werden. Unter einer CHT kommt es zu einer ovariellen Stromahyperplasie, der Kortex wird dicker und steifer, aber die Anzahl der Primordial- und Antralfollikel scheint unverändert [20, 
21]. Trans ${ }^{\star}$ Männern kann zur Fertilitätsprotektion die Kryokonservierung von Oozyten angeboten werden, dies bedarf allerdings einer hormonellen Stimulation, wie sie im Rahmen einer klassischen In-vitro-Fertilisation durchgeführt wird. Um den Einfluss der erhöhten Östrogenspiegel unter einer Stimulation $\mathrm{zu}$ minimieren, wäre auch eine zusätzliche Verabreichung eines Aromataseinhibitors wie Letrozol denkbar. Eine weitere Möglichkeit der Fertilitätsprotektion ist die Kryokonservierung von Ovargewebe. Diese für Fertilitätsprotektion bei malignen Erkrankungen etablierte $\mathrm{Me}$ thode ist allerdings mit der späteren Autotransplantation des Ovargewebes und nachfolgender Stimulation verbunden [22]. Ein In-vitro-Wachstum und eine nachfolgende Maturation von Oozyten aus kryokonserviertem Ovarialgewebe oder auch eine allogene oder Xenotransplantation sind eventuelle $\mathrm{Zu}$ kunftsperspektiven [23].

\section{Fallbeispiel 3}

Die 50-jährige Karin ist seit 2 Jahren bei uns in Behandlung. Sie erhält eine $\mathrm{CHT}$ in der Form von transdermalem $17 \beta$-Östradiol und Triptorelin 11,25 mg alle 12 Wochen. Karin möchte eine geschlechtsangleichende Operation durchführen lassen und wünscht zusätzlich eine Mammaaugmentation. Wir besprechen mit Karin die operativen Möglichkeiten und klären sie über die notwendigen Nachsorgen auf.

Nach den operativen Eingriffen bestellen wir Karin zur kurzfristigen Kontrolle der Östradiolspiegel ein, die Therapie mit GnRH-a konnte nach der Gonadektomie abgesetzt werden. Im weiteren Verlauf wird sich Karin jährlich für gynäkologische und endokrinologische Kontrollen bei uns vorstellen.

\section{Operative Maßnahmen}

Eine geschlechtsangleichende Operation sollte erst mit Volljährigkeit, also ab dem vollendeten 18. Lebensjahr und nach einem Jahr durchgehender CHT erfolgen. Die Mastektomie bei FTM stellt hier eine Ausnahme dar und kann bereits früher angedacht werden, der Zeitpunkt sollte vor allem der individuellen Situation angepasst werden [7, 8]. Falls eine Mammaaugmentation gewünscht wird, empfiehlt sich diese frühestens nach einem Jahr andauernder Östrogentherapie, um die natürliche Brustentwicklung zu ermöglichen. Wird eine genitale geschlechtsangleichende Operation angestrebt, stehen bei MTF verschiedene Techniken der Vaginalplastik mit Orchiektomie, Penektomie, Klitorisplastik und Labioplastik zur Verfügung [7, 8, 24]. Die geschlechtsangleichende Operation scheint einen positiven Effekt auf die Lebensqualität zu haben [25]. Nach Anlage einer Neovagina ist eine regelmäßige Dehnung der Vagina durch die Patientin selbst oder durch regelmäßigen penetrierenden Geschlechtsverkehr nötig, um die Weite und Tiefe der Neovagina zu erhalten. Für die Nachsorge gilt es außerdem zu beachten, dass die Prostata in situ verbleibt und dementsprechend PSA-Kontrollen nach den üblichen Vorgaben durchzuführen sind. Bei FTM können die geschlechtsangleichenden Operationen einerseits die Hysterektomie mit beidseitiger Salpingooophorektomie und andererseits die Kolpektomie, Metaidoioplastik, Skrotalplastik, Urethralplastik, Phalloplastik, das Einsetzen von Hodenprothesen und einer Penispumpe umfassen [7, 8, 24, 26]. Zur Bildung des Neopenis werden freie Lappentechniken angewandt, hierbei scheint sich mittlerweile der freie Unterarmlappen durchzusetzen [24]. Viele Trans ${ }^{\star}$ Männer entscheiden sich nur für eine Hysterektomie mit Adnexektomie und wünschen keinen Penisaufbau. Die häufigsten Beweggründe für eine genitale Rekonstruktion sind der Wunsch nach Miktion im Stehen und die Kohabitationsfähigkeit mit erhaltender Orgasmusfähigkeit. Der komplette Prozess einer genitalen Geschlechtsangleichung bei FTM kann mehr als ein Jahr in Anspruch nehmen und ist sehr häufig mit Komplikationen wie Harnwegsstenose und Fistelbildungen verbunden [26]. Alternativ gibt es mittlerweile ein mannigfaltiges Angebot von Penisprothesen $\mathrm{zu}$ unterschiedlichen Einsatzzwecken wie Miktion, Geschlechtsverkehr oder auch optischem Ausfüllen der Hose.

\section{Fazit für die Praxis}

Ein niedrigschwelliger Zugang zum Gesundheitssystem ist erstrebenswert und sollte sich nicht nur auf die diagnostischen und therapeutischen Maßnahmen in spezialisierten Zentren beschränken. Die Grundversorgung im niedergelassenen Bereich durch aufgeschlossene, engagierte KollegInnen ist für die PatientInnen von großer Bedeutung.

Eine zeitgerecht gestartete Pubertätsunterdrückung mittels GnRH-a kann die volle Entwicklung der als belastend empfundenen sekundären Geschlechtsmerkmale bei Trans*Jugendlichen verhindern. Eine gegengeschlechtliche Hormontherapie sollte möglichst physiologische Konzentrationen der Sexualsteroide des Wunschgeschlechts erreichen, um Risiken und Nebenwirkungen der Therapie zu minimieren. Die Hormontherapie sollte lebenslang bzw. bis zum Erreichen des natürlichen Menopausenalters fortgeführt werden. Dementsprechend ist eine lebenslange Betreuung auch unter Berücksichtigung einer präventiven Gesundheitsvorsorge vonnöten.

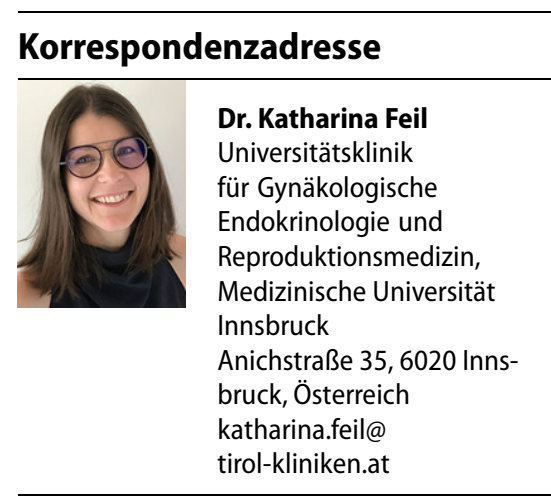

Funding. Open access funding provided by University of Innsbruck and Medical University of Innsbruck.

\section{Einhaltung ethischer Richtlinien}

Interessenkonflikt. K. Feil und B. Toth geben an, dass kein Interessenkonflikt besteht.

Für diesen Beitrag wurden von den Autorinnen keine Studien an Menschen oder Tieren durchgeführt. Für die aufgeführten Studien gelten die jeweils dort angegebenen ethischen Richtlinien. Für Bildmaterial oder anderweitige Angaben innerhalb des Manuskripts, über die Patienten zu identifizieren sind, liegt von 
ihnen und/oder ihren gesetzlichen Vertretern eine schriftliche Einwilligung vor.

Open Access Dieser Artikel wird unter der Creative Commons Namensnennung 4.0 International Lizenz veröffentlicht, welche die Nutzung, Vervielfältigung, Bearbeitung, Verbreitung und Wiedergabe in jeglichem Medium und Format erlaubt, sofern Sie den/die ursprünglichen Autor(en) und die Quelle ordnungsgemäß nennen, einen Link zur Creative Commons Lizenz beifügen und angeben, ob Änderungen vorgenommen wurden.

Die in diesem Artikel enthaltenen Bilder und sonstige Drittmaterial unterliegen ebenfalls der genannten Creative Commons Lizenz, sofern sich aus der Abbildungslegende nichts anderes ergibt. Sofern das betreffende Material nicht unter der genannten Creative Commons Lizenz steht und die betreffende Handlung nicht nach gesetzlichen Vorschriften erlaubt ist, ist für die oben aufgeführten Weiterverwendungen des Materials die Einwilligung des jeweiligen Rechteinhabers einzuholen.

Weitere Details zur Lizenz entnehmen Sie bitte de Lizenzinformation auf http://creativecommons.org/ licenses/by/4.0/deed.de.

\section{Literatur}

1. ICD-11 for Mortality and Morbidity Statistics (ICD11 MMS) 2018 version Available from: https://icd. who.int/browse11/l-m/en\#/http\%3a\%2f\%2fid. who.int\%2ficd\%2fentity\%2f90875286.

2. Collin L et al (2016) Prevalence of transgender depends on the "case" definition: a systematic review. JSex Med 13(4):613-626

3. Butler $G$ et al (2018) Assessment and support of children and adolescents with gender dysphoria. Arch Dis Child 103(7):631-636

4. Aitken $\mathrm{M}$ et al (2015) Evidence for an altered sex ratio in clinic-referred adolescents with gender dysphoria.J Sex Med 12(3):756-763

5. American Psychiatric Association (2013) Diagnostic and statistical manual of mental disorders. American Psychiatric Association, Washington, DC

6. Bundesministerium für Arbeit (2017) Gesundheit und Konsumentenschutz, Empfehlungen für den Behandlungsprozess bei Geschlechtsdysphorie bzw. Transsexualismus. http://www.bmgf.gv.at. Zugegriffen: 26.06.2020

7. Coleman E et al (2012) Standards of care for the health of transsexual, transgender, and gender-nonconforming people, version 7 . Int J Transgenderism 13(4):165-232

8. Hembree WC et al (2017) Endocrine treatment of gender-dysphoric/gender-incongruent persons: an endocrine society clinical practice guideline. JClin Endocrinol Metab 102(11):3869-3903

9. Thun-Hohenstein L, Riedl S, Bangerl HP, Fuchs M, Gottardi-Butturini E et al (2017) Empfehlungen für den Behandlungsprozess bei Geschlechtsdysphorie von Kindern und Jugendlichen nach der Klassifikation in der derzeit gültigen DSM- bzw. ICD-Fassung. Bundesministerium für Soziales, Gesundheit, Pflege und Konsumentenschutz

10. Winkler-Crepaz K, Feil K, Toth B et al (2019) Transgender in der Pubertät. Innsbrucker Erfahrungen. Transgender and puberty-experiences from Innsbruck. Internist Prax 61(03):440-449

11. Dobrolińska M et al (2019) Bone mineral density in transgender individuals after gonadectomy and long-term gender-affirming hormonal treatment. J Sex Med 16(9):1469-1477

12. Vlot MC et al (2017) Effect of pubertal suppression and cross-sex hormone therapy on bone turnover markers and bone mineral apparent density (BMAD) in transgender adolescents. Bone 95:11-19

13. betapharm (2020) (PRAC), S.d.E. Beschränkung der Anwendung von Cyproteron aufgrund Meningeom-Risikos. https://www. bfarm.de/SharedDocs/Risikoinformationen/ Pharmakovigilanz/DE/RHB/2020/rhb-cyproteron. pdf?_blob=publicationFile $\& v=2$. Zugegriffen: 28.06.2020

14. Velho l et al (2017) Effects of testosterone therapy on BMI, blood pressure, and laboratory profile of transgender men: a systematic review. Andrology 5(5):881-888

15. Rey RA, Grinspon RP (2020) Androgen treatment in adolescent males with hypogonadism. Am J Mens Health 14(3):1557988320922443

16. Ethics Committee of the American Society for Reproductive Medicine (2015) Access to fertility services by transgender persons: an ethics committee opinion. Fertil Steril 104(5):1111-1115

17. Chen D et al (2018) Attitudes toward fertility and reproductive health among transgender and gender-nonconforming adolescents. J Adolesc Health 63(1):62-68

18. Marsh C et al (2019) Low total motile sperm in transgender women seeking hormone therapy. J Assist Reprod Genet 36(8):1639-1648

19. Adeleye AJ et al (2019) Semen parameters among transgender women with a history of hormonal treatment. Urology 124:136-141

20. De Roo C et al (2019) Texture profile analysis reveals a stiffer ovarian cortex after testosteron therapy: a pilot study. J Assist Reprod Genet 36(9):1837-1843

21. Ikeda Ket al (2013) Excessive androgen exposure in female-to-male transsexual persons of reproductive age induces hyperplasia of the ovarian cortex and stroma but not polycystic ovary morphology. Hum Reprod 28(2):453-461

22. Dittrich $R$ et al (2018) Fertility preservation for patients with malignant disease. Guideline of the DGGG, DGU and DGRM (S2k-level, AWMF registry no. 015/082, november 2017)-recommendations and statements for girls and women. Geburtshilfe Frauenheilkd 78(6):567-584

23. Böttcher B, Toth B (2020) Update Fertilitätsprotektion bei Mädchen, Adoleszentinnen und Frauen. J Gynäkol Endokrinol AT. https://doi.org/10.1007/ s41974-020-00145-9

24. Sohn M, Rieger UM, Heß J (2017) Gender confirming surgery: Female-to-male and male-tofemale. Urologe A 56(10):1246-1255

25. Weinforth $\mathrm{G}$ et al (2019) Quality of life following male-to-female sex reassignment surgery. Dtsch Arztebl Int 116(15):253-260

26. Morrison SD, Chen ML, Crane CN (2017) An overview of female-to-male gender-confirming surgery. Nat Rev Urol 14(8):486-500

Hinweis des Verlags. Der Verlag bleibt in Hinblick auf geografische Zuordnungen und Gebietsbezeichnungen in veröffentlichten Karten und Institutsadressen neutral. 